Dig Dis 1995;13:391-392

\title{
Subject Index Vol. 13,1995
}

Abdominal trauma 56 Achalasia 56, 337 Acid secretion 25 Ambulatory esophageal manometry 145 Appendicitis 56

Balloon dilators 337

Bezoar 356

Bile acid diarrhoea 312

Bioartifícial liver 379

Bipolar electrocoagulation 68

Bougienage 337

Bowel infarction 3

Caffeine 239

Carcinogenesis 25, 205, 365 Cardiomyopathy 199 Cell adhesion molecules 322

Cholangiocarcinoma 39 Cholecystectomy 56 Cholera 190 -, epidemic 190 -, food contamination 190 Choleragen 190 Cholestyramine 312 Chronic intestinal pseudo-obstruction 163 Cirrhosis 136, 153 Cisapride 291 Collagenous colitis 301 Colon cancer screening 182 - $\quad$ surgery 56

Colonic mucosa, genetic changes 182

neoplasms 251 Colorectal cancer 39,365

-, inherited forms 182

neoplasms 322 Complications 251

-, cardiovascular 130 Cost effectiveness 56 Crohn's disease 39, 92

Diabetes 291

Diarrhea, choleragen-induced 190

Diet 365 Dilation 337 Domperidone 291 Duodenal ulcer 119 Dysphagia 337

Electrogastrography 275 Endoscopic therapy 68

ultrasound 39 Endoscopy 130 Enteral nutrition 92 Enterohepatic circulation 312 Environmental factors 365 Erythromycin 291 Esophageal cancer 39

motility disorders 145 Esophagus 337 Exchange transfusion 379 Extracorporeal liver perfusion 379

a-Fetoprotein 81 Fibrogenesis 205 Fulminant hepatic failure 199

Gastric cancer 39

dysrhythmia 275

emptying 275

motility 275

ulcer 119 Gastrin 25 Gastroduodenal hemorrhage 68

mucosal injury 251 Gastroesophageal reflux 56 Gastrointestinal bleeding 119

complications 119 Gastroparesis 291 Genetic factors 365

Heart disease 130 Heater probe 68 Hemochromatosis 205, 223 Hemodialysis 379 Hemofiltration 379 Hemoperfusion 379 Hepatic failure 379

- $\quad$ pharmacology 239 
391

Hepatocellular carcinoma 81 Hepatoma 81 Hepatotoxicity 205 Hereditary colorectal cancer 365

Histamine 25

Inflammation, intestinal 322 Inflammatory bowel disease 92,

322 Inguinal hernia 56 Injection agents 68 Intestinal ischemia 3 Iron 223

deficiency 223

metabolism 223

overload 205

Laparoscopy 56

-, indications 56

Lidocaine 239

Liver biopsy, complications 267

-, death rate 267

-, methodology, review 267

failure 379

transplantation 199 Lymphocytic colitis 301

Malabsorption 108 Malnutrition 92 Medication 356 Mercury dilators 337 Mesenteric circulation 3

emboli 3

thrombi 3 Metoclopramide 291 Microscopic colitis 301 Migrating motor complex 163

Molecular genetics, colorectal

cancer 182 Monoethylene-glycinexylidide

239 Myotomy 337

Neuroendocrine cells 25 Noncardiac chest pain 145 Nonsteroidal anti-inflammatory drugs

119,251

- $\quad$ - - enteropathy 251

Nutritional care 92

Outcomes research 39

Pancreatic cancer 39 Perforation 337 Pharmacobezoar 356 Plasma exchange 379

- perfusion 379

Portal hypertension treatment

153 Prostaglandins 251

Rehydration therapy, diarrhea 190

Selenohomocholic acid taurine

test 312 Serum zinc 136 Small bowel manometry 163

- $\quad$ intestinal motility 163

Stigmata 68

Structure 337

Total parenteral nutrition 92 Transjugular intrahepatic porto-

systemic shunt 153 Tropheryma whippelii 108 Tumor staging 39

Ulcerative colitis 92 Unexplained chest pain 145

Variceal hemorrhage 153 Vibrio cholerae 190 Viral hepatitis 223

Whipple's disease 108

Subject Index 